\title{
Nonlinear electromagnetic wave equations for superdense magnetized plasmas
}

\author{
Nitin Shukla a)], G. Brodin, M. Marklund, P. K. Shukla [b)], and L. Stenflo[c] \\ Department of Physics, Umeå University, SE-90187 Umeå,, SE-90187 Sweden
}

(Received 2 March 2009)

\begin{abstract}
By using the quantum hydrodynamic and Maxwell equations, we derive nonlinear electronmagnetohydrodynamic (MHD), Hall-MHD, and dust Hall-MHD equations for dense quantum magnetoplasmas. The nonlinear equations include the electromagnetic, the electron pressure gradient, as well as the quantum electron tunneling and electron spin forces. They are useful for investigating a number of wave phenomena including linear and nonlinear electromagnetic waves, as well as threedimensional electromagnetic wave turbulence spectra arising from the mode coupling processes in dense magnetoplasmas.
\end{abstract}

PACS numbers: 52.25.Dg, 52.27.Gr, 52.35.Mw, 71.10.Ca

\section{INTRODUCTION}

Superdense quantum plasmas are ubiquitous in compact astrophysical objects [1, 2, 3, 4] (e.g. the interior of white dwarf stars, magnetars, giant planetary interiors), and in the next generation intense laser-solid density plasma experiments 4, 5, 6, 7, 87. In dense plasmas the degenerate electrons follow Fermi-Dirac statistics, with quantum tunneling [9, 10, 11, 12, 13, 14, 15] and spin 16, 17, 18, 19, 20] forces due to the spread in the electron probability wave-function. The quantum statistical electron pressure and quantum forces produce wave dispersion at nanoscales. Accordingly, there have been a great deal of interest [10, 11, 15, 19, 20, 21] in investigating linear and nonlinear waves and structures at quantum scales in very dense plasmas.

It is well known that superdense plasmas in white dwarf stars and magnetars are strongly magnetized. Accordingly, the electron dynamics is greatly affected by the Lorentz force. Furthermore, there is a Bohr magnetization of electrons which have 1/2-spin. Thus, new electromagnetic wave theories (both linear and nonlinear) in dense magnetized plasmas have to be developed, accounting for the quantum statistical electron pressure, the quantum force associated with electron tunneling, and the Bohr electron magnetization due to the spin effect.

In this work, we derive nonlinear equations for electromagnetic waves in superdense quantum magnetoplasmas. Specifically, we focus on the electron-MHD, Hall-MHD, and dust Hall-MHD plasmas, and show how the density, fluid velocity, and magnetic fields are coupled in a non-trivial manner. The present equations are useful for studying numerically [22] the linear and nonlinear wave phenomena at quantum scales in superdense astrophysical plasmas.

\section{DERIVATION OF THE NONLINEAR EQUATIONS}

The governing nonlinear equations for electromagnetic waves in dense plasmas are the quantum hydrodynamic equations composed of the continuity equation

$$
\frac{\partial n_{j}}{\partial t}+\nabla \cdot\left(n_{j} \mathbf{u}_{j}\right)=0
$$

the electron momentum equation

$$
n_{e} m_{e}\left[\frac{\partial \mathbf{u}_{e}}{\partial t}+\mathbf{u}_{e} \cdot \nabla \mathbf{u}_{e}\right]=-n_{e} e\left[\mathbf{E}+\frac{1}{c} \mathbf{u}_{e} \times \mathbf{B}\right]-\nabla p_{e}+\mathbf{F}_{Q e}
$$

the Faraday law

$$
c \nabla \times \mathbf{E}=-\frac{\partial \mathbf{B}}{\partial t},
$$

the Maxwell equation including the magnetization spin current 


$$
\nabla \times \mathbf{B}=\frac{4 \pi}{c}\left(\mathbf{J}_{p}+\mathbf{J}_{m}\right)+\frac{1}{c} \frac{\partial \mathbf{E}}{\partial t}
$$

and the momentum equations

$$
n_{\sigma} m_{\sigma}\left[\frac{\partial \mathbf{u}_{\sigma}}{\partial t}+\mathbf{u}_{\sigma} \cdot \nabla \mathbf{u}_{\sigma}\right]=q_{\sigma} n_{\sigma}\left[\mathbf{E}+\frac{1}{c} \mathbf{u}_{\sigma} \times \mathbf{B}\right]-n_{\sigma} m_{\sigma} \nabla \phi_{g},
$$

where we have introduced the pressure for a non-relativistic degenerate electron gas

$$
p_{e}=\left(\frac{4 \pi^{2} \hbar^{2}}{5 m_{e}}\right)(3 / 8 \pi)^{2 / 3} n_{e}^{5 / 3}
$$

and the sum of the quantum tunneling and spin forces

$$
\mathbf{F}_{Q e}=\nabla\left(\frac{\nabla^{2} \sqrt{n_{e}}}{\sqrt{n_{e}}}\right)-n_{e} \mu_{B} \tanh (\xi) \nabla B
$$

The gravitational force is

$$
\nabla^{2} \phi_{g}=4 \pi G \sum_{\sigma=i, d} m_{\sigma} n_{\sigma}
$$

where $G$ is the gravitational constant.

In Eqs. (1)-(7), $n_{j}$ is the number density of the particle species $j$ ( $j$ equals $e$ for the electrons, $i$ for the ions, and $d$ for the dust grains), $\mathbf{u}_{e}$ is the electron fluid velocity, $\mathbf{u}_{\sigma}$ is the fluid velocity of the species $\sigma(\sigma=i, d$, the index $i$ and $d$ stand for the ions and charged dust grains), $m_{j}$ is the mass, $q_{\sigma}=Z_{i} e$ for the ions and $\epsilon Z_{d} e$ for the dust grains, $Z_{i}$ is the ion charge state, $e$ is the magnitude of the electron charge, $Z_{d}$ is the number of electron charges on a dust grain, $\epsilon=-1(+1)$ for negative (positive) dust, $c$ is the speed of light in vacuum, $\mu_{B}=e \hbar / 2 m_{e}$ is the Bohr magneton, $\hbar$ is the Planck constant divided by $2 \pi$, and $B=|\mathbf{B}|$. We have here introduced the plasma current density $\mathbf{J}_{p}=-e n_{e} \mathbf{u}_{e}+Z_{i} e n_{i} \mathbf{u}_{i}+\epsilon Z_{d} e n_{d} \mathbf{u}_{d}$ and the electron magnetization spin current density $\mathbf{J}_{m}=\nabla \times \mathbf{M}$, where the magnetization for dynamics on a time scale much slower than the spin precession frequency reads $\mathbf{M}=$ $n_{e} \mu_{B} \tanh (\xi) \hat{\mathbf{B}}$. Here $\tanh (\xi)=B_{1 / 2}(\xi), B_{1 / 2}$ is the Brillouin function with argument $1 / 2$ describing electrons of spin $1 / 2, \xi=\mu_{B} B / k_{B} T_{F e}, \hat{\mathbf{B}}=\mathbf{B} / B, k_{B}$ is the Boltzmann constant, and $T_{F e}$ is the Fermi electron temperature. We have assumed that the spin orientation has reached the thermodynamical equilibrium state in response to the magnetic field, which accounts for the $\tanh (\xi)$-factor. On a time scale shorter than the spin relaxation time scale, the individual electron spins are conserved, and thus $\tanh (\xi)$ can be taken as constant for an initially inhomogeneous plasma.

Let us first present the generalized nonlinear electron-MHD equations for dense plasmas. Here the ions and dust grains form the neutralizing background. The wave phenomena in the EMHD plasma will occur on a time scale much shorter than the ion/dust plasma and gyroperiods. In equilibrium, we have [23]

$$
n_{e 0}=Z_{i} n_{i 0}+\epsilon Z_{d} n_{d 0},
$$

where the subscript 0 stands for the unperturbed value.

The relevant electron-MHD equations are

$$
\frac{\partial n_{e}}{\partial t}+\nabla \cdot\left(n_{e} \mathbf{u}_{e}\right)=0
$$

the electron momentum equation (2), Faraday's law (3) and the electron fluid velocity given by 


$$
\mathbf{u}_{e}=\frac{\mathbf{J}_{m}}{e n_{e}}-\frac{c(\nabla \times \mathbf{B})}{4 \pi e n_{e}}+\frac{\partial \mathbf{E}}{e n_{e} \partial t} .
$$

We observe that the quantum tunneling and spin forces play a role if there are slight electron density and magnetic field inhomogeneities in dense plasmas.

Second, we derive the modified Hall-MHD equations in the presence of immobile dust grains. The Hall-MHD equations shall deal with the wave phenomena on a time scale larger than the electron gyroperiod. The relevant equations are the electron and ion continuity equations, the inertialess electron momentum equation

$$
\mathbf{E}+\frac{1}{c} \mathbf{u}_{e} \times \mathbf{B}+\frac{\nabla p_{e}}{n_{e} e}-\frac{\mathbf{F}_{Q e}}{n_{e} e}=0
$$

Faraday's law (3), the ion momentum equation

$$
n_{i} m_{i}\left[\frac{\partial \mathbf{u}_{i}}{\partial t}+\mathbf{u}_{i} \cdot \nabla \mathbf{u}_{i}\right]=Z_{i} e n_{i}\left[\mathbf{E}+\frac{1}{c} \mathbf{u}_{i} \times \mathbf{B}\right]-n_{i} m_{i} \nabla \phi_{g}
$$

with

$$
\nabla^{2} \phi_{g}=4 \pi G m_{i} n_{i}
$$

and the electron fluid velocity given by

$$
\mathbf{u}_{e}=\frac{Z_{i} n_{i} \mathbf{u}_{i}}{n_{e}}+\frac{\mathbf{J}_{m}}{e n_{e}}-\frac{c(\nabla \times \mathbf{B})}{4 \pi e n_{e}},
$$

where we have neglected the displacement current since the Hall-MHD plasma deals with electromagnetic waves whose phase velocity is much smaller than the speed of light in vacuum.

We now eliminate the electric field from (12) by using (11), obtaining

$$
n_{i} m_{i}\left[\frac{\partial \mathbf{u}_{i}}{\partial t}+\mathbf{u}_{i} \cdot \nabla \mathbf{u}_{i}\right]=Z_{i} e n_{i}\left[\frac{1}{c}\left(\mathbf{u}_{i}-\mathbf{u}_{e}\right) \times \mathbf{B}-\frac{\nabla p_{e}}{n_{e} e}+\frac{\mathbf{F}_{Q e}}{n_{e} e}\right]-n_{i} m_{i} \nabla \phi_{g} .
$$

Furthermore, eliminating $\mathbf{u}_{e}$ from (15) by using (14), we have

$$
n_{i} m_{i}\left[\frac{\partial \mathbf{u}_{i}}{\partial t}+\mathbf{u}_{i} \cdot \nabla \mathbf{u}_{i}\right]=Z_{i} e n_{i}\left\{\frac{1}{c}\left[\frac{\epsilon Z_{d} n_{d}}{n_{e}} \mathbf{u}_{i}-\frac{\mathbf{J}_{m}}{e n_{e}}+\frac{c(\nabla \times \mathbf{B})}{4 \pi e n_{e}}\right] \times \mathbf{B}-\frac{\nabla p_{e}}{n_{e} e}+\frac{\mathbf{F}_{Q e}}{n_{e} e}\right\}-n_{i} m_{i} \nabla \phi_{g}
$$

where $n_{e}=Z_{i} n_{i}+\epsilon Z_{d} n_{d}$ and $Z_{d} n_{d}$ is constant.

Finally, by using (14) we can eliminate $\mathbf{E}$ from (3), obtaining

$$
\frac{\partial \mathbf{B}}{\partial t}=\nabla \times\left[\frac{Z_{i} n_{i} \mathbf{u}_{i} \times \mathbf{B}}{n_{e}}+\frac{\mathbf{J}_{m} \times \mathbf{B}}{e n_{e}}-\frac{c}{4 \pi e n_{e}}(\nabla \times \mathbf{B}) \times \mathbf{B}\right]
$$

The ion continuity equation, Eqs. (16) and (17), together with (13) and the quasi-neutrality condition $n_{i 1}=n_{e 1}$, where $n_{e 1, i 1} \ll n_{e, i 0}$ are the desired generalized nonlinear Hall-MHD dense self-gravitating plasma with immobile charged dust grains. show a nontrivial linear and nonlinear coupling between the density, ion fluid velocity and magnetic fields fluctuations. They describe the dynamics of a broad range of electromagnetic waves in dense quantum plasmas.

Third, we consider a dense magnetoplasma composed of inertialess electrons and ions, as well inertial dust grains. The dust mass density $n_{d} m_{d}$ is supposed to be much larger than the ion mass density $n_{i} m_{i}$. The relevant dust Hall-MHD equations, valid for the low-frequency (in comparison with the ion gyrofrequency) electromagnetic waves, are then composed of the dust continuity equation 


$$
\frac{\partial n_{d}}{\partial t}+\nabla \cdot\left(n_{d} \mathbf{u}_{d}\right)=0
$$

the inertialess electron and ion momentum equations, which are combined with the dust momentum equation (in which the electric field is eliminated by using the inertialess electron and ion momentum equation)

$$
n_{d} m_{d} \frac{d \mathbf{u}_{d}}{d t}=\left(\frac{1}{4 \pi} \nabla \times \mathbf{B}-\frac{1}{c} \mathbf{J}_{m}\right) \times \mathbf{B}-\frac{\epsilon Z_{d} n_{d} \nabla p_{e}}{n_{e}}+\frac{\epsilon Z_{d} n_{d} \mathbf{F}_{Q e}}{n_{e}}-n_{d} m_{d} \nabla \phi_{g}
$$

with

$$
\nabla^{2} \phi_{g} \approx 4 \pi G n_{d} m_{d}
$$

together with Faraday's law (3). Here $d / d t=(\partial / \partial t)+\mathbf{u}_{d} \cdot \nabla$. In deriving (19) we have used the modified Ampère's law [e.g. Eq.(4) without the displacement current] in view of the low-phase velocity (in comparison with $c$ ) electromagnetic waves.

From Faraday's law (3) and the dust momentum equation, we obtain

$$
\frac{\partial \mathbf{B}}{\partial t}=\nabla \times\left(\mathbf{u}_{d} \times \mathbf{B}\right)-\frac{c m_{d}}{q_{d}} \nabla \times \frac{d \mathbf{u}_{d}}{d t} .
$$

Equations (18), (19) and (21) with $n_{e} \approx Z_{d} n_{d} \gg Z_{i} n_{i 0}$ are the desired dust Hall-MHD equations in dense magnetized plasmas. These equations govern the dynamics of the modified coupled dust-cyclotron, dust acoustic and dispersive dust Alfvén waves. The dispersion arises due to the quantum electron tunneling and the finite frequency (in comparison with the dust gyrofrequency) or the dust skin effect.

\section{SUMMARY}

In this paper, we have derived the nonlinear equations for electromagnetic waves in dense self-gravitating magnetoplasmas. For this purpose, we have used the generalized quantum hydrodynamical and Maxwell's equations, and obtained three sets of nonlinear equations, which exhibit the non-trivial linear and nonlinear couplings between the plasma number density, fluid velocity, and magnetic field fluctuations. The present set of nonlinear equations should be used to investigate numerically the dynamics of obliquely (against the external magnetic field direction) propagating modified electron whistlers with the generalized electron-MHD plasma model, as well as the modified fast and slow modes and dispersive electromagnetic ion-cyclotron-kinetic Alfvén waves [24, 25] within the generalized Hall-MHD plasma model, and the modified coupled dust-cyclotron-dust acoustic and dispersive dust Alfvén waves within the dust Hall-MHD plasma model. The obtained results would then provide valuable information on multidimensional electromagnetic fluctuation spectra at nanoscales, which may appear in the interior of white dwarf stars and in magnetars.

This work was partially supported by the Deutsche Forschungsgemeinschaft (Bonn) through the project SH21/3-1 of the Forschergruppe FOR 1048, as well as by the European Research Council under Contract No. 204059-QPQV, and by the Swedish Research Council under Contracts No. 2005-4967 and No. 2007-4422.

[a)] Also at GoLP/Instituto de Plasmas Fusão Nuclear, Instituto Superior Técnico, Universidade Técnica do Lisboa, 1049-001 Lisboa, Portugal. E-mail: nitshukla@yahoo.com

[b)] Also at Department of Physics, Umeå University, SE-90187 Umeå, Sweden; Scottish Universities Physics Alliance (SUPA), Department of Physics, University of Strathclyde, Glasgow G4 ONG, U. K.; GoLP/Instituto de Plasmas Fusão Nuclear, Instituto Superior Técnico, Universidade Técnica do Lisboa, 1049-001 Lisboa, Portugal; School of Physics, University of KwaZulu-Natal, 4000 Durban, South Africa; Max-Planck Institut für Extraterrestrische Physik und Plasmaphysik, D-85740 Garching, Germany. E-mail: ps@tp4.rub.de

[c)] Also at Department of Physics, Linköping University, SE-58183 Linkp̈ing, Sweden. E-mail: lennart.stenflo@physics.umu.se 
[1] H. M. van Horn, Science 252, 384 (1991); R. Schlickeiser and P. K. Shukla, Astrophys. J. 599, L57 (2003).

[2] A. K. Harding and D. Lai, Phys. Rep. 69, 2631 (2006).

[3] G. Chabrier, F. Douchin, and A. Y. Potekhin, J. Phys. Condens. Matter 14, 9133 (2002); G. Chabrier, D. Saumon, and A. Y. Potekhin, J. Phys. A: Math. Gen. 39, 4411 (2006); A. Y. Potekhin, G. Chabrier, D. Lai, W. C. G. Ho, and M. van Adelsberg, ibid. 39, 4453 (2006)..

[4] M. Marklund and P. K. Shukla, Phys. Mod. Phys. 78, 591 (2006).

[5] V. Berezhiani, D. D. Tskhakaya, and P. K. Shukla, Phys. Rev. A 46, 6608 (1992).

[6] S. Eliezer, P. Norreys, J. T. Mendonça, and K. Lancaster, Phys. Plasmas 12, 052115 (2005).

[7] S. H. Glenzer,O. L. Landen, P. Neumayer et al., Phys. Rev. Lett. 98, 065002 (2007).

[8] V. M. Malkin, N. J. Fisch, and J. S. Wurtele, Phys. Rev E. 75, 026404 (2007).

[9] C. L. Gardner and C. Ringhofer, Phys. Rev. E 53, 157 (1996).

[10] G. Manfredi and F. Haas, Phys. Rev. B 64, 075316 (2001); F. Haas, L. G. Garcia, J. Goedert, and G. Manfredi, Phys. Plasmas 10, 3858 (2003); F. Haas, Phys. Plasmas 12, 062117 (2005).

[11] G. Manfredi, Fields Inst. Commun. 46, 263 (2005); N. Crouseilles, P. A. Hervieux, and G. Manfredi, Phys. Rev. B 78, $155412(2008)$.

[12] P. K. Shukla, Phys. Lett. A 352, 242 (2006); D. Jovanovic and R. Fedele, ibid. 364, 304 (2007).

[13] A. Serbeto, J. T. Mendonça, K. H. Tsui, and R. Bonifacio, Phys. Plasmas 15, 013110 (2008).

[14] M. Marklund, G. Brodin, L. Stenflo, and C. S. Liu, Europhys. Lett. 84, 17006 (2008).

[15] P. K. Shukla and B. Eliasson, Phys. Rev. Lett.96, 245001 (2006); ibid. 99, 096401 (2007).

[16] M. Marklund and G. Brodin, Phys. Rev. Lett. 98, 025001 (2007).

[17] G. Brodin and M. Marklund, Phys. Plasmas 14, 112107 (2007); New J. Phys. 9, 277 (2007); Phys. Rev. E 76, 055403(R).

[18] A. P. Misra, Phys. Plasmas 14, 064501 (2007).

[19] G. Brodin, M. Marklund, M.Zamanian, A. Ericsson, and P. L. Mana, Phys. Rev. Lett. 101, 245002 (2008).

[20] P. K. Shukla, Nature Phys. 5, 92 (2009).

[21] D. Shaikh and P. K. Shukla, Phys. Rev. Lett. 99, 125002 (2007).

[22] D. Shaikh and P. K. Shukla, Phys. Rev. Lett. 102, 045004 (2009).

[23] P. K. Shukla and B. Eliasson, Rev. Mod. Phys. 81, 25 (2009).

[24] P. K. Shukla, I. Kourakis, and L. Stenflo, Phys. Rev. Lett. 94, 119501 (2005).

[25] P. K. Shukla, I. Kourakis, and L. Stenflo, Phys. Plasmas 12, 024501 (2005). 